QUARTERLY OF APPLIED MATHEMATICS

VOLUME LXX, NUMBER 2

JUNE 2012, PAGES 285-297

S 0033-569X(2012)01267-8

Article electronically published on February 3, 2012

\title{
GLOBAL WEAK SOLUTIONS FOR A PERIODIC TWO-COMPONENT HUNTER-SAXTON SYSTEM
}

\author{
BY
}

CHUNXIA GUAN (Institut Franco-Chinois de l'Energie Nucléaire)

AND

ZHAOYANG YIN (Department of Mathematics, Sun Yat-sen University, 510275 Guangzhou, China)

Abstract. This paper is concerned with global existence of weak solutions for a periodic two-component Hunter-Saxton system. We first derive global existence for strong solutions to the system with smooth approximate initial data. Then we show that the limit of approximate solutions is a global weak solution of the two-component HunterSaxton system.

1. Introduction. In this paper we consider the following periodic two-component Hunter-Saxton system:

$$
\begin{cases}u_{t x x}+2 u_{x} u_{x x}+u u_{x x x}-\rho \rho_{x}=0, & t>0, x \in \mathbb{R}, \\ \rho_{t}+(\rho u)_{x}=0, & t>0, x \in \mathbb{R}, \\ \rho(t, x+1)=\rho(t, x), & t \geq 0, x \in \mathbb{R}, \\ u(t, x+1)=u(t, x), & t \geq 0, x \in \mathbb{R}, \\ u(0, x)=u_{0}(x), & x \in \mathbb{R} \\ \rho(0, x)=\rho_{0}(x), & x \in \mathbb{R} .\end{cases}
$$

This set of equations arises in the short-wave (or high-frequency) limits

$$
(t, x) \rightarrow(\varepsilon t, \varepsilon x), \quad \varepsilon \rightarrow 0
$$

of the two-component Camassa-Holm equation $(2 \mathrm{CH})$ [4, 11, 25.

$$
\begin{gathered}
u_{t}-u_{t x x}+3 u u_{x}=2 u_{x} u_{x x}+u u_{x x x}+k \rho \rho_{x} \\
\rho_{t}+(\rho u)_{x}=0
\end{gathered}
$$

Received July 29, 2010.

2010 Mathematics Subject Classification. Primary 35G25, 35L05.

Key words and phrases. Periodic two-component Hunter-Saxton system, weak solutions, global existence, approximate solutions.

E-mail address: guanchunxia123@163.com

E-mail address: mcsyzy@mail.sysu.edu.cn

(C) 2012 Brown University Reverts to public domain 28 years from publication 
derived from the Green-Naghdi equations, which are approximations to the governing equations for water waves. The main motivation for seeking and studying such systems lies in capturing nonlinear phenomena such as wave breaking and traveling waves which are not exhibited by small-amplitude models [6, 25. The system (1.1) is a particular case of the Gurevich-Zybin system describing the dynamics in a model of nondissipative dark matter (cf. 23, 25] and the references therein).

For $\rho \equiv 0$, the system (1.1) reduces to the Hunter-Saxton equation [15] which describes the propagation of weakly nonlinear orientation waves in a massive nematic liquid crystal director field. Here, $u(t, x)$ describes the director field of a nematic liquid crystal, $x$ is a space variable in a reference frame moving with the linearized wave velocity, and $t$ is a slow time variable. More precisely, the orientation of the molecules is described by the field of unit vectors $(\cos u(t, x), \sin u(t, x))$ [15, 25].

The Hunter-Saxton equation also arises in a different physical context as the highfrequency limit [10, 16] of the Camassa-Holm equation - a model equation for shallow water waves [3, 14] and a re-expression of the geodesic flow on the diffeomorphism group of the circle [5] with a bi-Hamiltonian structure [13] which is completely integrable 7]. The Hunter-Saxton equation also has a bi-Hamiltonian structure [15, 24] and is completely integrable [1, 16], and it is also the Euler equation for the geodesic flow on the quotient space of the infinite-dimensional group $D(\mathbb{S})$ of orientation-preserving diffeomorphisms of the unit circle $\mathbb{S}=\mathbb{R} / \mathbb{Z}$ modulo the subgroup of rotations $\operatorname{Rot}(\mathbb{S})$ equipped with the $H^{1}$ right-invariant metric [19, 20, 25].

The initial value problem for the Hunter-Saxton equation on the line (nonperiodic case) was studied by Hunter and Saxton in [15. Using the method of characteristics, they showed that smooth solutions exist locally and break down in finite time. The occurrence of blow-up can be interpreted physically as the phenomenon by which waves that propagate away from the perturbation "knock" the director field out of its unperturbed state [15]. The initial value problem for the periodic Hunter-Saxton equation was studied in 26. Using the Kato theorem, the author obtained that this equation has solutions for initial data $u_{0} \in H^{s}(\mathbb{S}), s>\frac{3}{2}$, and showed that all the nonconstant solutions blow up in finite time [26].

Recently, global dissipative and conservative weak solutions for the Hunter-Saxton equation on the line were investigated extensively, cf. [2, 8, 17, 18, 27, 28, 29.

For $\rho \not \equiv 0$, peakon solutions of the system (1.1) were analyzed in [4. Moreover, the Cauchy problem of the system (1.1) was discussed in 25, 22. However, the existence of global weak solutions to the system (1.1) has not been studied yet. The aim of this paper is to present a global existence result of weak solutions to the system (1.1).

The main result of this paper is to give the existence of a global-in-time weak solution $z=\left(\begin{array}{l}u \\ \rho\end{array}\right)$ to the problem (1.1) with the initial $z_{0}=\left(\begin{array}{c}u_{0} \\ \rho_{0}\end{array}\right) \in W^{1, \infty}(\mathbb{S}) \times L^{\infty}(\mathbb{S})$. Before giving the precise statement of the main result, we first introduce the definition of a weak solution to the problem (1.1).

DeFinition 1.1. $z=\left(\begin{array}{c}u \\ \rho\end{array}\right) \in H^{1}(\mathbb{S}) \times L^{2}(\mathbb{S})$ is said to be an admissible weak solution to the problem (1.1) if

$$
z(t, x) \in L_{\mathrm{loc}}^{\infty}\left((0, \infty) ; H^{1}(\mathbb{S}) \times L^{2}(\mathbb{S})\right)
$$


satisfies the system (1.1) and $z(t, \cdot) \rightarrow z_{0}$ as $t \rightarrow 0^{+}$in the sense of distributions on $\mathbb{R}_{+} \times \mathbb{R}$. Moreover,

$$
\left\|u_{x}(t, \cdot)\right\|_{L^{2}(\mathbb{S})}^{2}+\|\rho(t, \cdot)\|_{L^{2}(\mathbb{S})}^{2} \leq\left\|u_{0, x}\right\|_{L^{2}(\mathbb{S})}^{2}+\left\|\rho_{0}\right\|_{L^{2}(\mathbb{S})}^{2} .
$$

The main result of this paper can be stated as follows:

Theorem 1.1. Let $z_{0}=\left(\begin{array}{c}u_{0} \\ \rho_{0}\end{array}\right) \in W^{1, \infty}(\mathbb{S}) \times L^{\infty}(\mathbb{S})$. If there exists $\alpha>0$ such that $\rho_{0}(x) \geq \alpha$ for a.e. $x \in \mathbb{S}$, then the system (1.1) has an admissible weak solution

$$
z=\left(\begin{array}{c}
u \\
\rho
\end{array}\right) \in C\left(\mathbb{R}_{+} ; H^{1}(\mathbb{S}) \times L^{2}(\mathbb{S})\right) \cap L_{\mathrm{loc}}^{\infty}\left(\mathbb{R}_{+} ; H^{1}(\mathbb{S}) \times L^{2}(\mathbb{S})\right)
$$

in the sense of Definition 1.1. Furthermore,

$$
u \in L_{\mathrm{loc}}^{\infty}\left(\mathbb{R}_{+} ; W^{1, \infty}(\mathbb{S})\right) \text { and } \rho \in L_{\mathrm{loc}}^{\infty}\left(\mathbb{R}_{+} ; L^{\infty}(\mathbb{S})\right) .
$$

REMARK 1.1. If there exists $\alpha<0$ such that $\rho_{0}(x) \leq \alpha$ for a.e. $x \in \mathbb{S}$, then the conclusions in Theorem 1.1 also hold.

The paper is organized as follows. In Section 2, we recall some useful lemmas and obtain the global existence of strong solutions to (1.1) with smooth approximate initial data. Moreover, we derive some a priori estimates on these strong solutions. In Section 3 , acquiring the precompactness of approximate solutions, we prove the existence of the global weak solution to (1.1).

2. Preliminaries. We provide now the framework in which we shall reformulate the system (1.1). Integrating both sides of the first equation in (1.1) with respect to $x$, we obtain

$$
u_{t x}+u u_{x x}+\frac{1}{2} u_{x}^{2}-\frac{1}{2} \rho^{2}=-a(t)
$$

where

$$
a(t)=\frac{1}{2} \int_{\mathbb{S}}\left(\rho^{2}+u_{x}^{2}\right) d x .
$$

A simple calculation 25] yields

$$
\frac{d}{d t} a(t)=0 .
$$

So let us henceforth write $a:=a(0)$. Therefore,

$$
u_{t x}+u u_{x x}=\frac{1}{2} \rho^{2}-\frac{1}{2} u_{x}^{2}-a
$$

is a valid reformulation of the first equation in (1.1). Integrating once more in $x$, we get

$$
u_{t}+u u_{x}=\partial_{x}^{-1}\left(\frac{1}{2} \rho^{2}+\frac{1}{2} u_{x}^{2}-a\right)+h(t),
$$


where $\partial_{x}^{-1} f(x):=\int_{0}^{x} f(y) d y$ and $h(t):[0, \infty) \rightarrow \mathbb{R}$ is an arbitrary continuous function. If choosing $h(t) \equiv 0$, then we get the following system:

$$
\begin{cases}u_{t}+u u_{x}=\partial_{x}^{-1}\left(\frac{1}{2} \rho^{2}+\frac{1}{2} u_{x}^{2}-a\right), & t>0, x \in \mathbb{R}, \\ \rho_{t}+(\rho u)_{x}=0, & t>0, x \in \mathbb{R}, \\ u(t, x+1)=u(t, x), & t \geq 0, x \in \mathbb{R}, \\ \rho(t, x+1)=\rho(t, x), & t \geq 0, x \in \mathbb{R}, \\ u(0, x)=u_{0}(x), & x \in \mathbb{R}, \\ \rho(0, x)=\rho_{0}(x), & x \in \mathbb{R},\end{cases}
$$

where $\partial_{x}^{-1} f(x):=\int_{0}^{x} f(y) d y$. If $z=\left(\begin{array}{c}u \\ \rho\end{array}\right) \in H^{1}(\mathbb{S}) \times L^{2}(\mathbb{S})$ is a weak solution of $(2.2)$, then $z$ is a weak solution of (1.1). To obtain the existence of global weak solutions to (1.1), we study (2.2) henceforth.

Lemma 2.1 (22]). Let $z_{0}=\left(\begin{array}{c}u_{0} \\ \rho_{0}\end{array}\right) \in H^{s}(\mathbb{S}) \times H^{s-1}(\mathbb{S}), s \geq 2$. If $\rho_{0}(x) \neq 0$ for all $x \in \mathbb{S}$, then the corresponding strong solution $z=\left(\begin{array}{l}u \\ \rho\end{array}\right)$ to $(2.2)$ exists globally in time, i.e., $z \in C\left(\mathbb{R}_{+} ; H^{s}(\mathbb{S}) \times H^{s-1}(\mathbb{S})\right) \cap C^{1}\left(\mathbb{R}_{+} ; H^{s-1}(\mathbb{S}) \times H^{s-2}(\mathbb{S})\right)$. Moreover, there exists $\beta>0$ such that for all $t \in \mathbb{R}$,

$$
\left\|u_{x}(t, \cdot)\right\|_{L^{\infty}(\mathbb{S})} \leq \frac{1}{2 \beta}\left(1+\left\|\rho_{0}\right\|_{L^{\infty}(\mathbb{S})}^{2}+\left\|u_{0, x}\right\|_{L^{\infty}(\mathbb{S})}^{2}\right) e^{(2 a+1) t} \equiv C_{1}(t)
$$

and

$$
\|\rho(t, \cdot)\|_{L^{\infty}(\mathbb{S})} \leq e^{C_{1}(t) t}\left\|\rho_{0}\right\|_{L^{\infty}(\mathbb{S})} \equiv C_{2}(t),
$$

where $\beta=\inf _{x \in \mathbb{S}}\left|\rho_{0}(x)\right|$. Furthermore,

$$
\int_{\mathbb{S}}\left(\rho_{0}^{2}(t, x)+u_{x}^{2}(t, x)\right) d x=\int_{\mathbb{S}}\left(\rho_{0}^{2}+u_{0, x}^{2}\right) d x, \quad \forall t \in \mathbb{R}_{+} .
$$

Lemma 2.2. Assume $z_{0}=\left(\begin{array}{l}u_{0} \\ \rho_{0}\end{array}\right) \in H^{s}(\mathbb{S}) \times H^{s-1}(\mathbb{S}), s \geq 2$, and $\rho_{0}(x) \neq 0$ for all $x \in \mathbb{S}$, Let $z=\left(\begin{array}{l}u \\ \rho\end{array}\right)$ be the corresponding global strong solution to (2.2) which is guaranteed by Lemma 2.1. Then we have

$$
\|u(t, \cdot)\|_{L^{2}(\mathbb{S})}^{2} \leq e^{t}\left(\left\|u_{0}\right\|_{L^{2}(\mathbb{S})}^{2}+4 a^{2}\right)
$$

and

$$
\|u(t, \cdot)\|_{L^{\infty}(\mathbb{S})}^{2} \leq \frac{13}{12}\left\{e^{t}\left(\left\|u_{0}\right\|_{L^{2}(\mathbb{S})}^{2}+4 a^{2}\right)+\left\|u_{0, x}\right\|_{L^{2}(\mathbb{S})}^{2}+\left\|\rho_{0}\right\|_{L^{2}(\mathbb{S})}^{2}\right\} .
$$

Proof. In view of (2.3) and equation (2.2), we have that for any $t \geq 0$,

$$
\begin{aligned}
\frac{d}{d t} \int_{\mathbb{S}} u(t, x)^{2} d x & =2 \int_{\mathbb{S}} u \partial_{x}^{-1}\left(\frac{1}{2} u_{x}^{2}+\frac{1}{2} \rho^{2}-a\right) d x \\
& \leq \int_{\mathbb{S}} u^{2} d x+\frac{1}{4} \int_{\mathbb{S}}\left(\int_{0}^{x}\left(u_{y}^{2}+\rho^{2}-2 a\right) d y\right)^{2} d x \\
& \leq \int_{\mathbb{S}} u(t, x)^{2} d x+4 a^{2}
\end{aligned}
$$

By Gronwall's inequality we get

$$
\int_{\mathbb{S}} u(t, x)^{2} d x \leq e^{t}\left(\int_{\mathbb{S}} u_{0}(x)^{2} d x+4 a^{2}\right) .
$$


This proves (2.4). In view of (2.3)-(2.4) and Sobolev's imbedding theorem, we obtain

$$
\|u(t, \cdot)\|_{L^{\infty}(\mathbb{S})}^{2} \leq \frac{13}{12}\left\{e^{t}\left(\left\|u_{0}\right\|_{L_{\mathbb{S}}^{2}}^{2}+4 a^{2}\right)+\left\|u_{0, x}\right\|_{L^{2}(\mathbb{S})}^{2}+\left\|\rho_{0}\right\|_{L^{2}(\mathbb{S})}^{2}\right\} .
$$

This completes the proof of the lemma.

Lemma 2.3 (Appendix C of [21]). Let $X$ be a separable reflexive Banach space, and let $f^{n}$ be bounded in $L^{\infty}(0, T ; X)$ for some $T \in(0, \infty)$. We assume that $f^{n} \in C([0, T] ; Y)$ where $Y$ is a Banach space such that $X \hookrightarrow Y, Y^{\prime}$ is separable and dense in $X^{\prime}$. Furthermore, $\left(\phi, f^{n}(t)\right)_{Y^{\prime} \times Y}$ is uniformly continuous in $t \in[0, T]$ and uniformly in $n \geq 1$. Then $f^{n}$ is relatively compact in $C^{w}([0, T] ; X)$, the space of continuous functions from $[0, T]$ with values in $X$ when the latter space is equipped with its weak topology.

REMARK 2.1. If the conditions satisfied by $f^{n}$ in Lemma 2.3 are replaced with

$$
f^{n} \in L^{\infty}(0, T ; X), \partial_{t} f^{n} \in L^{p}(0, T ; Y) \text { for some } p \in(1, \infty)
$$

and

$$
\left\|f^{n}\right\|_{L^{\infty}(0, T ; X)},\left\|\partial_{t} f^{n}\right\|_{L^{p}(0, T ; Y)} \leq C, \quad \forall n \geq 1,
$$

then the conclusion of Lemma 2.3 holds true.

3. The existence of global weak solution. In this section, we first prove the existence of approximate solutions. With the basic energy estimate in Section 2, we are ready to obtain the necessary compactness of the approximate solutions $z^{n}(t, x)$. Acquiring the precompactness of approximate solutions, we prove the existence of the global weak solution to the system (2.2).

Let $z_{0}=\left(\begin{array}{c}u_{0} \\ \rho_{0}\end{array}\right) \in W^{1, \infty}(\mathbb{S}) \times L^{\infty}(\mathbb{S})$, and there exists $\alpha>0$ such that $\rho_{0}(x) \geq \alpha>0$ for a.e. $x \in \mathbb{S}$. Define $z_{0}^{n}:=\left(\begin{array}{c}\phi_{n} * u_{0} \\ \phi_{n} * \rho_{0}\end{array}\right)=\left(\begin{array}{c}u_{0}^{n} \\ \rho_{0}^{n}\end{array}\right) \in H^{s}(\mathbb{S}) \times H^{s-1}(\mathbb{S}), s \geq 3$, for $n \geq 1$; here $\left\{\phi_{n}\right\}_{n \geq 1}$ are the mollifiers

$$
\phi_{n}(x):=\left(\int_{\mathbb{R}} \phi(\xi) d \xi\right)^{-1} n \phi(n x), \quad x \in \mathbb{R}, \quad n \geq 1,
$$

where $\phi \in C_{c}^{\infty}(\mathbb{R})$ is defined by

$$
\phi(x)= \begin{cases}e^{1 /\left(x^{2}-1\right)}, & |x|<1 \\ 0, & |x| \geq 1 .\end{cases}
$$

In view of $\rho_{0}(x) \geq \alpha>0$, for a.e. $x \in \mathbb{R}$ and $\phi_{n}(x) \geq 0$, we have

$$
\rho_{0}^{n}(x)=\phi_{n} * \rho_{0}(x) \geq \alpha \int_{\mathbb{R}} \phi_{n}(y) d y=\alpha>0, \quad \forall x \in \mathbb{R} .
$$

Clearly, we also have

$$
u_{0}^{n} \rightarrow u_{0} \quad \text { in } H^{1}(\mathbb{S}), \quad \rho_{0}^{n} \rightarrow \rho_{0} \text { in } L^{2}(\mathbb{S}), \quad \text { as } n \rightarrow \infty
$$

and

$$
\left\|u_{0}^{n}\right\|_{L^{2}(\mathbb{S})} \leq\left\|u_{0}\right\|_{L^{2}(\mathbb{S})},\left\|u_{0, x}^{n}\right\|_{L^{2}(\mathbb{S})} \leq\left\|u_{0, x}\right\|_{L^{2}(\mathbb{S})},\left\|\rho_{0}^{n}\right\|_{L^{2}(\mathbb{S})} \leq\left\|\rho_{0}\right\|_{L^{2}(\mathbb{S})} .
$$

Thus, we obtain the corresponding solution $z^{n} \in C\left(\mathbb{R}_{+} ; H^{s}(\mathbb{S}) \times H^{s-1}(\mathbb{S})\right)$ to the system (2.2) with initial data $z_{0}^{n}$ by Lemma 2.1 . 
For given $z_{0}=\left(\begin{array}{c}u_{0} \\ \rho_{0}\end{array}\right) \in W^{1, \infty}(\mathbb{S}) \times L^{\infty}(\mathbb{S})$, we set

$$
a^{n}:=\frac{1}{2}\left(\left\|u_{0, x}^{n}\right\|_{L^{2}(\mathbb{S})}^{2}+\left\|\rho_{0}^{n}\right\|_{L^{2}(\mathbb{S})}^{2}\right), a_{0}:=\frac{1}{2}\left(\left\|u_{0, x}\right\|_{L^{2}(\mathbb{S})}^{2}+\left\|\rho_{0}\right\|_{L^{2}(\mathbb{S})}^{2}\right) .
$$

Then we have the following remark.

REMARK 3.1. By Lemmas 2.1-2.2 and (3.2), we have

$$
a^{n} \rightarrow a_{0}, \text { as } n \rightarrow \infty
$$

and

$$
\left\|u_{x}^{n}(t, \cdot)\right\|_{L^{2}(\mathbb{S})}^{2}+\left\|\rho^{n}(t, \cdot)\right\|_{L^{2}(\mathbb{S})}^{2}=2 a^{n} \leq 2 a_{0}, \quad \forall t \in \mathbb{R}_{+} .
$$

Moreover, by Sobolev's imbedding theorem, we get

$$
\left\|u^{n}(t, \cdot)\right\|_{L^{\infty}(\mathbb{S})}^{2} \leq \frac{13}{12}\left\{e^{t}\left(\left\|u_{0}\right\|_{L^{2}(\mathbb{S})}^{2}+4 a^{2}\right)+\left\|u_{0, x}\right\|_{L^{2}(\mathbb{S})}^{2}+\left\|\rho_{0}\right\|_{L^{2}(\mathbb{S})}^{2}\right\} .
$$

Furthermore, by Lemma 2.1, we obtain

$$
\left\|u_{x}^{n}(t, \cdot)\right\|_{L^{\infty}(\mathbb{S})} \leq C_{1}(t), \quad \text { and }\left\|\rho^{n}(t, \cdot)\right\|_{L^{\infty}(\mathbb{S})} \leq C_{2}(t), \quad \forall t \in \mathbb{R}_{+}
$$

where $C_{1}(t)$ and $C_{2}(t)$ are given in Lemma 2.1.

Lemma 3.1. For any fixed $T>0$, there exist a subsequence $\left\{z^{n_{k}}(t, x)\right\}$ of the sequence $\left\{z^{n}(t, x)\right\}$ and some function $z(t, x) \in L^{\infty}\left((0, T) ; H^{1}(\mathbb{S}) \times L^{2}(\mathbb{S})\right)$ such that

$$
z^{n_{k}} \rightarrow z \quad \text { in } H^{1}((0, T) \times \mathbb{S}) \times L^{2}((0, T) \times \mathbb{S}) \text { as } n_{k} \rightarrow \infty, \forall T>0
$$

and

$$
u^{n_{k}} \rightarrow u \text { uniformly on each compact subset of } \mathbb{R}_{+} \times \mathbb{R} \text { as } n_{k} \rightarrow \infty \text {. }
$$

Proof. It follows from Lemmas 2.1 and 2.2 and Remark 3.1 that for any fixed $T>0$, $\left\{z^{n}(t, x)\right\}$ is uniformly bounded in $L^{\infty}\left((0, T) ; H^{1}(\mathbb{S}) \times L^{2}(\mathbb{S})\right)$. Also, by $(2.2)$ we have $\left\{\partial_{t} u^{n}(t, x)\right\}$ is uniformly bounded in $L^{2}((0, T) \times \mathbb{S})$. By the classical Lions-Aubin lemma, there exist $u \in L^{\infty}\left((0, T) ; H^{1}(\mathbb{S})\right)$ and a subsequence $\left\{u_{\epsilon_{k}}(t, x)\right\}$ such that $\left\{u_{\epsilon_{k}}(t, x)\right\}$ is weakly compact in $L^{\infty}\left((0, T), H^{1}(\mathbb{S})\right)$ and $\left\{u_{\epsilon_{k}}(t, x)\right\}$ converges to $u(t, x)$ uniformly on each compact subset of $\mathbb{R}_{+} \times \mathbb{R}$ as $k \rightarrow \infty$. Moreover, $u(t, x)$ is a continuous function.

Furthermore, from Lemma 2.1, we can easily obtain that $\rho^{n}$ is uniformly bounded in the space $L^{2}((0, T) \times \mathbb{S})$. Therefore, there exist $z=\left(\begin{array}{c}u \\ \rho\end{array}\right) \in L^{\infty}\left((0, T) ; H^{1}(\mathbb{S}) \times L^{2}(\mathbb{S})\right)$ and a subsequence $\left\{z^{n_{k}}(t, x)\right\}$ such that

$$
z^{n_{k}} \rightarrow z \quad \text { in } H^{1}((0, T) \times \mathbb{S}) \times L^{2}((0, T) \times \mathbb{S}) \text { as } n_{k} \rightarrow \infty .
$$

This completes the proof of the lemma.

REMARK 3.2. By Lemmas 2.1 and 2.2 and the above argument, there exists a subsequence of $\left\{\left(u_{x}^{n}\right)^{2},\left(\rho^{n}\right)^{2}\right\}$, denoted again by $\left\{\left(u_{x}^{n_{k}}\right)^{2},\left(\rho^{n_{k}}\right)^{2}\right\}$, converging weakly in $L_{\text {loc }}^{p}\left(\mathbb{R}_{+} \times \mathbb{R}\right)$, where $1<p<\infty$; i.e., there exist $\overline{u_{x}^{2}} \in L_{\text {loc }}^{p}\left(\mathbb{R}_{+} \times \mathbb{R}\right)$ and $\overline{\rho^{2}} \in L_{\text {loc }}^{p}\left(\mathbb{R}_{+} \times \mathbb{R}\right)$ such that

$$
\left(u_{x}^{n_{k}}\right)^{2} \rightarrow \overline{u_{x}^{2}} \text { and }\left(\rho^{n_{k}}\right)^{2} \rightarrow \overline{\rho^{2}} \text { in } L_{\mathrm{loc}}^{p}\left(\mathbb{R}_{+} \times \mathbb{R}\right) .
$$

Moreover, we have

$$
\begin{gathered}
u_{x}^{n_{k}} \rightarrow u_{x} \text { in } L_{\mathrm{loc}}^{p}\left(\mathbb{R}_{+} \times \mathbb{R}\right) \text { and } u_{x}^{n_{k}}-^{\star} u_{x} \text { in } L^{\infty}\left(\mathbb{R}_{+} ; L^{2}(\mathbb{S})\right), \\
\rho^{n_{k}} \rightarrow \rho \text { in } L_{\mathrm{loc}}^{p}\left(\mathbb{R}_{+} \times \mathbb{R}\right) \text { and } \rho^{n_{k}}{{ }^{\star}}^{\star} \rho \text { in } L^{\infty}\left(\mathbb{R}_{+} ; L^{2}(\mathbb{S})\right) .
\end{gathered}
$$


Furthermore, we have

$$
u_{x}^{2}(t, x) \leq \overline{u_{x}^{2}}(t, x) \quad \rho^{2}(t, x) \leq \overline{\rho^{2}}(t, x) \quad \text { a.e. on } \mathbb{R}_{+} \times \mathbb{R} .
$$

In view of (3.6), we have

$$
\left\|u_{x}(t, \cdot)\right\|_{L^{\infty}(\mathbb{S})} \leq C_{1}(t), \quad\|\rho(t, \cdot)\|_{L^{\infty}(\mathbb{S})} \leq C_{2}(t), \quad \forall t \in \mathbb{R}_{+},
$$

where $C_{1}(t)$ and $C_{2}(t)$ are given in Lemma 2.1.

LEMma 3.2. There hold

$$
\lim _{t \rightarrow 0^{+}} \int_{\mathbb{S}} u_{x}^{2}(t, x) d x=\lim _{t \rightarrow 0^{+}} \int_{\mathbb{S}} \overline{u_{x}^{2}}(t, x)=\int_{\mathbb{S}} u_{0, x}^{2}(x) d x
$$

and

$$
\lim _{t \rightarrow 0^{+}} \int_{\mathbb{S}} \rho^{2}(t, x) d x=\lim _{t \rightarrow 0^{+}} \int_{\mathbb{S}} \overline{\rho^{2}}(t, x) d x=\int_{\mathbb{S}} \rho_{0}^{2}(x) d x .
$$

Proof. By Lemma 3.1, for any $T>0$, we have $u^{n} \in L^{\infty}\left((0, T) ; H^{1}(\mathbb{S})\right), u_{t}^{n}$ are uniformly bounded in $L^{\infty}\left((0, T) ; L^{2}(\mathbb{S})\right)$ and $u^{n} \in C\left([0, T] ; H^{1}(\mathbb{S})\right)$ by Lemma 2.1. Then in view of Lemma 2.3, Remark 2.1, and the proof of Lemma 3.1, we have $\left\{u^{n}\right\}$ contains a subsequence, denoted again by $\left\{u^{n_{k}}\right\}$, converging weakly in $H^{1}(\mathbb{S})$ uniformly in $t \in(0, T)$. The limit function is $u$. This implies that $u$ is weakly continuous from $(0, T)$ into $H^{1}(\mathbb{S})$, i.e.,

$$
u \in C^{w}\left([0, T] ; H^{1}(\mathbb{S})\right) .
$$

Similarly, as $\rho^{n} \in L^{\infty}\left((0, T) ; L^{2}(\mathbb{S})\right)$, in view of (3.4)-(3.5), we get that for all $t \in(0, T)$,

$$
\begin{aligned}
\left\|\rho_{t}^{n}(t, \cdot)\right\|_{H^{-1}(\mathbb{S})} & =\sup _{\|\phi\|_{H^{1}(\mathbb{S})}=1} \int_{\mathbb{S}}\left(u^{n} \rho^{n}\right) \phi_{x} d x \leq\left\|u^{n} \rho^{n}\right\|_{L^{2}(\mathbb{S})} \\
& \leq C(T)\left\|\rho_{0}\right\|_{L^{2}(\mathbb{S})},
\end{aligned}
$$

where $(C(t))^{2}=e^{t}\left(\left\|u_{0}\right\|_{L^{2}(\mathbb{S})}^{2}+4 a_{0}^{2}\right)+\left\|u_{0, x}\right\|_{L^{2}(\mathbb{S})}^{2}+\left\|\rho_{0}\right\|_{L^{2}(\mathbb{S})}^{2}$. This shows that $\rho_{t}^{n}$ is uniformly bounded in $L^{\infty}\left((0, T) ; H^{-1}(\mathbb{S})\right)$. Then by Lemma 2.3 and Remark 2.1 , we have $\left\{\rho^{n}\right\}$ contains a subsequence, denoted again by $\left\{\rho^{n_{k}}\right\}$, converging weakly in $L^{2}(\mathbb{S})$ uniformly in $t$. The limit function is $\rho$. This implies that $\rho$ is weakly continuous from $(0, T)$ into $L^{2}(\mathbb{S})$, i.e.,

$$
\rho \in C^{w}\left([0, T] ; L^{2}(\mathbb{S})\right) .
$$

Then by (3.13)-(3.14), we get

$$
\rho(t, \cdot) \rightarrow \rho_{0} \text { and } u_{x}(t, \cdot) \rightarrow u_{0, x} \quad \text { in } L^{2}(\mathbb{S}) \quad \text { as } t \rightarrow 0^{+} .
$$

Thus, we have

$$
\liminf _{t \rightarrow 0^{+}} \int_{\mathbb{S}} \rho^{2}(t, x) d x \geq \int_{\mathbb{S}} \rho_{0}^{2}(x) d x
$$

and

$$
\liminf _{t \rightarrow 0^{+}} \int_{\mathbb{S}} u_{x}^{2}(t, x) d x \geq \int_{\mathbb{S}} u_{0, x}^{2}(x) d x .
$$


Therefore, we deduce

$$
\begin{aligned}
& \liminf _{t \rightarrow 0^{+}} \int_{\mathbb{S}} u_{x}^{2}(t, x) d x+\rho^{2}(t, x) d x \\
& \quad \geq \liminf _{t \rightarrow 0^{+}} \int_{\mathbb{S}} u_{x}^{2}(t, x) d x+\liminf _{t \rightarrow 0^{+}} \int_{\mathbb{S}} \rho^{2}(t, x) d x \\
& \quad \geq \int_{\mathbb{S}} u_{0, x}^{2}(x)+\rho_{0}^{2}(x) d x .
\end{aligned}
$$

Moreover, from Lemma 2.1 and (3.2), we infer

$$
\begin{aligned}
& \int_{\mathbb{S}}(\left.\overline{u_{x}^{2}}(t, x)+\overline{\rho^{2}}(t, x)\right) d x \\
& \quad \leq \liminf _{n_{k} \rightarrow \infty} \int_{\mathbb{S}}\left(\left(u_{x}^{n_{k}}\right)^{2}(t, x)+\left(\rho^{n_{k}}\right)^{2}(t, x)\right) d x \\
& \quad=\liminf _{n_{k} \rightarrow \infty} \int_{\mathbb{S}}\left(\left(u_{0, x}^{n_{k}}\right)^{2}(x)+\left(\rho_{0}^{n_{k}}\right)^{2}(x)\right) d x \\
& \quad=\int_{\mathbb{S}}\left(u_{0, x}^{2}(x)+\rho_{0}^{2}(x)\right) d x .
\end{aligned}
$$

Thus, we obtain

$$
\limsup _{t \rightarrow 0^{+}} \int_{\mathbb{S}}\left(\overline{u_{x}^{2}}(t, x)+\overline{\rho^{2}}(t, x)\right) d x \leq \int_{\mathbb{S}}\left(u_{0, x}^{2}(x)+\rho_{0}^{2}(x)\right) d x .
$$

In view of (3.9), (3.15)-(3.16), we get (3.11) and (3.12). This completes the proof of the lemma.

Multiplying equation (2.1) and the second equation in (2.2) by $2 u_{x}^{n_{k}}$ and $2 \rho^{n_{k}}$, respectively, in view of (3.6), we get

$$
\frac{\partial}{\partial t}\left(u_{x}^{n_{k}}\right)^{2}+\frac{\partial}{\partial x}\left(u^{n_{k}}\left(u_{x}^{n_{k}}\right)^{2}\right)=\left(\rho^{n_{k}}\right)^{2} u_{x}^{n_{k}}-2 a^{n_{k}} n u_{x}^{n_{k}}
$$

and

$$
\frac{\partial}{\partial t}\left(\rho^{n_{k}}\right)^{2}+\frac{\partial}{\partial x}\left(u^{n_{k}}\left(\rho^{n_{k}}\right)^{2}\right)+u_{x}^{n_{k}}\left(\rho^{n_{k}}\right)^{2}=0 .
$$

Adding the above two equalities and letting $n_{k} \rightarrow \infty$, in view of Remark 3.2, we have

$$
\frac{\partial}{\partial t}\left(\overline{u_{x}^{2}}+\overline{\rho^{2}}\right)+\frac{\partial}{\partial x}\left(u\left[\overline{u_{x}^{2}}+\overline{\rho^{2}}\right]\right)=-2 a_{0} u_{x},
$$

in the sense of distributions on $((0, T) \times \mathbb{R})$.

On the other hand, by Lemma 3.1 and Remark 3.2, in view of equation (2.1) and the second equation in (2.2), letting $n_{k} \rightarrow \infty$, we obtain

$$
u_{t x}+\left(u u_{x}\right)_{x}=\frac{1}{2} \overline{u_{x}^{2}}+\frac{1}{2} \overline{\rho^{2}}-a_{0}
$$

and

$$
\rho_{t}+(u \rho)_{x}=0,
$$

in the sense of distributions on $((0, T) \times \mathbb{R})$. From (3.18) and (3.19), we have the following lemma. 
LEMma 3.3. There hold

$$
\frac{\partial\left(u_{x}\right)^{2}}{\partial t}+\frac{\partial}{\partial x}\left(u\left(u_{x}\right)^{2}\right)=\left(\overline{u_{x}^{2}}-u_{x}^{2}\right) u_{x}+\overline{\rho^{2}} u_{x}-2 a_{0} u_{x}
$$

and

$$
\frac{\partial \rho^{2}}{\partial t}+\frac{\partial}{\partial x}\left(u \rho^{2}\right)=-u_{x} \rho^{2}
$$

in the sense of distributions on $((0, T) \times \mathbb{R})$.

Proof. Denote $u_{n, x}(t, x):=\left(\left(u_{x}(t, \cdot) * \phi_{n}\right)(x)\right.$ and $\rho_{n}(t, x):=\left(\rho(t, \cdot) * \phi_{n}\right)(x)$. According to Lemma II.1 of [9], it follows from (3.18)-(3.19) that $u_{n, x}$ and $\rho_{n}$ solve

$$
\frac{\partial u_{n, x}}{\partial t}+u \frac{\partial u_{n, x}}{\partial x}=\left(-u_{x}^{2}+\frac{1}{2}\left(\overline{u_{x}^{2}}+\overline{\rho^{2}}\right)-a_{0}\right) * \phi_{n}+\tau_{n}
$$

and

$$
\frac{\partial \rho_{n}}{\partial t}+u \frac{\partial \rho_{n}}{\partial x}+\left(u_{x} \rho\right) * \phi_{n}=\sigma_{n},
$$

where the errors $\tau_{n}$, and $\sigma_{n}$ tend to zero in $L_{\text {loc }}^{1}\left(\mathbb{R}_{+} \times \mathbb{R}\right)$. Multiplying (3.22) and (3.23) by $2 u_{n, x}$ and $2 \rho_{n}$, respectively, we get

$$
\frac{\partial\left(u_{n, x}\right)^{2}}{\partial t}+\frac{\partial}{\partial x}\left(u u_{n, x}^{2}\right)=\left(\left(\overline{u_{x}^{2}}+\overline{\rho^{2}}-2 u_{x}^{2}-2 a_{0}\right) * \phi_{n}\right) u_{n, x}+u_{x} u_{n, x}^{2}+2 \tau_{n} u_{n, x}
$$

and

$$
\frac{\partial\left(\rho_{n}\right)^{2}}{\partial t}+\frac{\partial}{\partial x}\left(u \rho_{n}^{2}\right)=u_{x} \rho_{n}^{2}-2\left(\left(u_{x} \rho\right) * \phi_{n}\right) \rho_{n}+2 \tau_{n} \rho_{n} .
$$

We send $n \rightarrow \infty$ in (3.24) and (3.25) to obtain (3.20) and (3.21).

Adding (3.20) and (3.21), we deduce

$$
\frac{\partial\left(u_{x}^{2}+\rho^{2}\right)}{\partial t}+\frac{\partial}{\partial x}\left(u\left(u_{x}^{2}+\rho^{2}\right)\right)=\left(\overline{u_{x}^{2}}-u_{x}^{2}+\overline{\rho^{2}}-\rho^{2}-2 a_{0}\right) u_{x} .
$$

Next, we give the main lemma of this section.

Lemma 3.4. There hold

$$
\overline{u_{x}^{2}}(t, x)=u_{x}^{2}(t, x) \text { and } \overline{\rho^{2}}(t, x)=\rho^{2}(t, x) \text { a.e. on }(0, T) \times \mathbb{R} .
$$

Proof. Subtracting (3.26) from (3.17) and integrating the obtained equality over $(\varepsilon, t) \times$ $\mathbb{S}$ give

$$
\begin{aligned}
\int_{\mathbb{S}}\left(\overline{u_{x}^{2}}\right. & \left.-u_{x}^{2}+\overline{\rho^{2}}-\rho^{2}\right)(t, x) d x \\
\leq & -\int_{\varepsilon}^{t} \int_{\mathbb{S}}\left(\overline{u_{x}^{2}}-u_{x}^{2}+\overline{\rho^{2}}-\rho^{2}\right) u_{x}(t, x) d x d s \\
& +\int_{\mathbb{S}}\left(\overline{u_{x}^{2}}(\varepsilon, x)-u_{x}^{2}(\varepsilon, x)+\overline{\rho^{2}}(\varepsilon, x)-\rho^{2}(\varepsilon, x)\right) d x,
\end{aligned}
$$

for almost all $t \in(0, T)$. Letting $\varepsilon \rightarrow 0$ and using Lemma 3.2 and (3.10), we get

$$
\int_{\mathbb{S}}\left(\overline{u_{x}^{2}}-u_{x}^{2}+\overline{\rho^{2}}-\rho^{2}\right)(t, x) d x \leq C_{1}(T) \int_{\varepsilon}^{t} \int_{\mathbb{S}}\left(\overline{u_{x}^{2}}-u_{x}^{2}+\overline{\rho^{2}}-\rho^{2}\right) d x d s .
$$


Using Gronwall's inequality and Lemma 3.2, we obtain

$$
\int_{\mathbb{S}}\left(\overline{u_{x}^{2}}-u_{x}^{2}+\overline{\rho^{2}}-\bar{\rho}^{2}\right)(t, x) d x \leq 0
$$

By (3.9), we deduce

$$
0 \leq \int_{\mathbb{S}}\left(\overline{u_{x}^{2}}-u_{x}^{2}+\overline{\rho^{2}}-\rho^{2}\right)(t, x) d x \leq 0,
$$

which yields

$$
\int_{\mathbb{S}}\left(\overline{u_{x}^{2}}-u_{x}^{2}\right)(t, x) d x=\int_{\mathbb{S}}\left(\overline{\rho^{2}}-\rho^{2}\right)(t, x) d x=0 .
$$

In view of $u_{x}^{n}, u_{x}$ and $\rho^{n}, \rho$ being periodic with respect to $x$, we deduce that (3.27) holds.

To prove our main theorem, we need the following lemma.

Lemma 3.5 ([12]). Let $U$ be the bounded domain in $\mathbb{R}^{n}$. Assume that the sequence $\left\{f_{k}\right\}_{k=1}$ is bounded in $L^{1}\left(U ; \mathbb{R}^{m}\right)$. Then there exist a subsequence $\left\{f_{k_{j}}\right\}_{j=1} \subset\left\{f_{k}\right\}_{k=1}$ and, for a.e. $x \in U$, a Borel probability measure $\nu_{x}$ on $\mathbb{R}^{m}$ such that for any $F \in C\left(\mathbb{R}^{m}\right)$ we have

$$
F\left(f_{k_{j}}\right) \rightarrow^{*} \bar{F} \text { in } L^{\infty}(U),
$$

where $\bar{F}(x)=\int_{\mathbb{R}^{m}} F(y) d \nu_{x}$ for a.e. $x \in U$. Moreover, if $\nu_{x}$ is a unit point mass for a.e. $x \in U$, then $\left\{f_{k_{j}}\right\} \rightarrow f$ in $L^{2}\left(U ; \mathbb{R}^{m}\right)$, here $f(x)=\int_{\mathbb{R}^{m}} y d \nu_{x}$.

By (3.6) or (3.10), for any $T>0$ and $a<b,\left\{u_{x}^{n}\right\}$ and $\left\{\rho^{n}\right\}$ are uniformly bounded on $(0, T) \times(a, b)$. Using Lemma 3.5, there exist a subsequence $\left\{u_{x}^{n_{k}}, \rho^{n_{k}}\right\}_{k=1}^{\infty} \subset\left\{u_{x}^{n}, \rho^{n}\right\}_{n=1}^{\infty}$ for a.e. $(t, x) \in(0, T) \times(a, b)$ and two Borel probability measures $\mu_{(t, x)}, \nu_{(t, x)}$ on $\mathbb{R}$ such that for each $F \in C(\mathbb{R})$ we have

$$
F\left(u_{x}^{n_{k}}\right) \rightarrow^{*} \bar{F}_{1}, \quad F\left(\rho^{n_{k}}\right) \rightarrow^{*} \bar{F}_{2} \quad \text { in } L^{\infty}((0, T) \times(a, b)),
$$

where $\bar{F}_{1}=\int_{\mathbb{R}} F(y) d \mu_{(t, x)}$ and $\bar{F}_{2}=\int_{\mathbb{R}} F(y) d \nu_{(t, x)}$ for a.e. $x \in(0, T) \times(a, b)$. By Lemma 3.4, we have that $\mu_{(t, x)}=\delta_{u_{x}(t, x)}$ and $\nu_{(t, x)}=\delta_{\rho(t, x)}$ for a.e. $x \in(0, T) \times(a, b)$. Then, in view of Lemma 3.5 and from the arbitrariness of $T, a, b$, we obtain

$$
u_{x}^{n_{k}} \rightarrow u_{x}, \quad \rho^{n_{k}} \rightarrow \rho \quad \text { in } \quad L_{\mathrm{loc}}^{2}\left(\mathbb{R}_{+} \times \mathbb{R}\right) .
$$

With the above preparations, we now conclude the proof of the Theorem 1.1. Let $z=\left(\begin{array}{l}u \\ p\end{array}\right)$ be the limit of the approximate solutions $z^{n_{k}}$ as $k \rightarrow \infty$. It then follows from Lemma 2.1, Lemma 3.1, and Remark 3.2 that $u \in C([0,+\infty) \times \mathbb{R}) \cap L_{\text {loc }}^{\infty}\left(\mathbb{R}_{+}, H^{1}(\mathbb{S})\right)$ and $\rho \in L^{\infty}\left(\mathbb{R}_{+}, L^{2}(\mathbb{S})\right)$ hold. From Lemmas 3.1 and 3.4, in view of Remark 3.1 and (3.28), we have that $u^{n_{k}} \rightarrow u,\left(u^{n_{k}}\right)^{2} \rightarrow u^{2}$, and

$$
\partial_{x}^{-1}\left(\frac{1}{2}\left(u_{x}^{n_{k}}\right)^{2}+\frac{1}{2}\left(\rho^{n_{k}}\right)^{2}-a^{n_{k}}\right) \rightarrow \partial_{x}^{-1}\left(\frac{1}{2} u_{x}^{2}+\frac{1}{2} \rho^{2}-a_{0}\right)
$$

in the sense of distributions on $\mathbb{R}_{+} \times \mathbb{R}$. This shows that $z$ satisfies (2.2) in the sense of distributions on $\mathbb{R}_{+} \times \mathbb{R}$. By (3.4), (3.10) and the above estimates, we deduce that $z=\left(\begin{array}{l}u \\ \rho\end{array}\right)$ is an admissible weak solution to (1.1). 
From Lemma 3.2, we have $z \in C^{w}\left(\mathbb{R}_{+} ; H^{1}(\mathbb{S}) \times L^{2}(\mathbb{S})\right)$. Note that $u \in C\left((0, \infty) ; L^{2}(\mathbb{S})\right)$. In order to conclude that $z \in C\left(\mathbb{R}_{+} ; H^{1}(\mathbb{S}) \times L^{2}(\mathbb{S})\right)$, it is enough to show that $E(t)=$ $\int_{\mathbb{S}}\left(u_{x}^{2}(t)+\rho^{2}(t)\right) d x$ is conserved in time. Indeed, if this holds, then

$$
\begin{aligned}
\| z(t)- & z(s) \|_{H^{1}(\mathbb{S}) \times L^{2}(\mathbb{S})}^{2} \\
= & \|u(t)-u(s)\|_{L^{2}(\mathbb{S})}^{2}+\left\|u_{x}(t)-u_{x}(s)\right\|_{L^{2}(\mathbb{S})}^{2}+\|\rho(t)-\rho(s)\|_{L^{2}(\mathbb{S})}^{2} \\
= & \|u(t)-u(s)\|_{L^{2}(\mathbb{S})}^{2}+\left\|u_{x}(t)\right\|_{L^{2}(\mathbb{S})}^{2}-2\left(u_{x}(t), u_{x}(s)\right)_{L^{2}(\mathbb{S})}+\left\|u_{x}(s)\right\|_{L^{2}(\mathbb{S})}^{2} \\
& +\|\rho(t)\|_{L^{2}(\mathbb{S})}^{2}-2(\rho(t), \rho(s))_{L^{2}(\mathbb{S})}+\|\rho(s)\|_{L^{2}(\mathbb{S})}^{2} \\
= & \|u(t)-u(s)\|_{L^{2}(\mathbb{S})}^{2}+2 E(t)-2\left(u_{x}(t), u_{x}(s)\right)_{L^{2}(\mathbb{S})}-2(\rho(t), \rho(s))_{L^{2}(\mathbb{S})} .
\end{aligned}
$$

Since $\|u(t)-u(s)\|_{L^{2}(\mathbb{S})}^{2} \rightarrow 0$ and

$$
\left(u_{x}(t), u_{x}(s)\right)_{L^{2}(\mathbb{S})}+(\rho(t), \rho(s))_{L^{2}(\mathbb{S})} \rightarrow\left\|u_{x}(t)\right\|_{L^{2}(\mathbb{S})}^{2}+\|\rho(t)\|_{L^{2}(\mathbb{S})}^{2}=E(t)
$$

as $s \rightarrow t$, we deduce $z \in C\left(\mathbb{R}_{+} ; H^{1}(\mathbb{S}) \times L^{2}(\mathbb{S})\right)$.

Denote $u_{n, x}(t, x):=\left(u_{x}(t, \cdot) * \phi_{n}\right)(x)$ and $\rho_{n}(t, x):=\left(\rho(t, \cdot) * \phi_{n}\right)(x)$. According to Lemma II.1 of [9], we may deduce from (3.24)-(3.25) and (3.28) that $u_{n, x}$ and $\rho_{n}$ solve

$$
\frac{\partial\left(u_{n, x}\right)^{2}}{\partial t}+\frac{\partial}{\partial x}\left(u u_{n, x}^{2}\right)=\left(\left(-u_{x}^{2}+\rho^{2}-2 a_{0}\right) * \phi_{n}\right) u_{n, x}+u_{x} u_{n, x}^{2}+2 \tau_{n} u_{n, x}
$$

and

$$
\frac{\partial\left(\rho_{n}\right)^{2}}{\partial t}+\frac{\partial}{\partial x}\left(u \rho_{n}^{2}\right)=u_{x} \rho_{n}^{2}-2\left(\left(u_{x} \rho\right) * \phi_{n}\right) \rho_{n}+2 \tau_{n} \rho_{n},
$$

where the errors $\tau_{n}$ and $\sigma_{n}$ tend to zero in $L^{1}(\mathbb{S})$. Adding (3.29) and (3.30), and then integrating by parts, we infer that

$$
\begin{aligned}
\frac{d}{d t} \int_{\mathbb{S}} & \left(u_{n, x}^{2}+\rho_{n}^{2}\right) d x \\
= & \int_{\mathbb{S}}\left(\left(\left(-u_{x}^{2}+\rho^{2}\right) * \phi_{n}\right) u_{n, x}+u_{x} u_{n, x}^{2}\right) d x+\int_{\mathbb{S}}\left(u_{x} \rho_{n}^{2}-2\left(\left(u_{x} \rho\right) * \phi_{n}\right) \rho_{n}\right) d x \\
& +\int_{\mathbb{S}} 2\left(\tau_{n} u_{n, x}+\tau_{n} \rho_{n}\right) d x .
\end{aligned}
$$

Note that for fixed $T>0, u, u_{x}$ and $\rho$ are bounded in $(0, T) \times \mathbb{S}$. An application of Lebesgue's dominated convergence theorem yields

$$
\frac{d}{d t} \int_{\mathbb{R}}\left(u_{x}^{2}+\rho^{2}\right) d x=0
$$

This completes the proof of Theorem 1.1.

Acknowledgments. This work was partially supported by NNSFC (No. 10971235), RFDP (No. 200805580014), NCET-08-0579 and the key project of Sun Yat-sen University. 


\section{REFERENCES}

[1] R. Beals, D. Sattinger and J. Szmigielski, Inverse scattering solutions of the Hunter-Saxton equations, Appl. Anal., 78 (2001), 255-269. MR1883537 (2002m:35187)

[2] A. Bressan and A. Constantin, Global solutions of the Hunter-Saxton equation, SIAM J. Math. Anal., 37 (2005), 996-1026. MR2191785 (2006j:35203)

[3] R. Camassa and D. Holm, An integrable shallow water equation with peaked solitons, Phys. Rev. Lett., 71 (1993), 1661-1664. MR.1234453 (94f:35121)

[4] A. Constantin and R. I. Ivanov, On an integrable two-component Camass-Holm shallow water system, Phys. Lett. A, 372 (2008), 7129-7132. MR2474608 (2009m:35418)

[5] A. Constantin and B. Kolev, On the geometric approach to the motion of inertial mechanical systems, J. Phys. A, 35 (2002), R51-R79. MR1930889 (2003g:37138)

[6] A. Constantin and D. Lannes, The hydrodynamical relevance of the Camassa-Holm and DegasperisProcesi equations, Arch. Rat. Mech. Anal., 1992 (2009), 165-186. MR2481064(2010f:35334)

[7] A. Constantin and H. McKean, A shallow water equation on the circle, Comm. Pure Appl. Math., 52 (1999), 949-982. MR:1686969 (2000m:37146)

[8] C. M. Dafermos, Continuous solutions for balance laws, Ricerche di Matematica, 55 (2006), 79-91. MR2248164 (2007h:35222)

[9] R. J. DiPerna and P. L. Lions, Ordinary differential equations, transport theory and Sobolev space, Invent. Math., 98 (1989), 511-547. MR1022305 (90j:34004)

[10] H. H. Dai and M. Pavlov, Transformations for the Camassa-Holm equation, its high-frequency limit and the Sinh-Gordon equation, J. P. Soc. Japan, 67 (1998), 3655-3657. MR.1677529

[11] J. Escher, O. Lechtenfeld and Z. Yin, Well-posedness and blow-up phenomena for the 2component Camassa-Holm equation, Discrete Contin. Dyn. Syst., 19 (2007), 493-513. MR2335761 $(2008 \mathrm{j}: 35154)$

[12] L. Evans, Weak Convergence Methods for Nonlinear Partial Differential Equations, CBMS Regional Conference Series in Mathematics, vol. 74, American Mathematical Society, Rhode Island, 1990. MR 1034481 (91a:35009)

[13] A. Fokas, and B. Fuchssteiner, Symplectic structures, their Bäcklund transformations and hereditary symmetries, Phys. D, 4 (1981/1982), 47-66. MR636470(84j:58046)

[14] R. Johnson, Camassa-Holm, Korteweg-de Vries and related models for water waves, J. Fluid. Mech., 457 (2002), 63-82. MR:1894796 (2003b:76026)

[15] J. Hunter and R. Saxton, Dynamics of director fields, SIAM J. Appl. Math., 51 (1991), 1498-1521. MR.1135995(93a:76005)

[16] J. Hunter and Y. Zheng, On a completely integrable nonlinear hyperbolic variational equation, Phys. D, 79 (1994), 361-386. MR1306466 (96e:35166)

[17] J. Hunter and Y. Zheng, On a nonlinear hyperbolic variational equation: I, Global existence of weak solutions, Arch. Rat. Mech. Anal., 129 (1995), 305-353. MR.1361013 (96m:35215)

[18] J. Hunter and Y. Zheng, On a nonlinear hyperbolic variational equation: II, The zero-viscosity and dispersion limits, Arch. Rat. Mech. Anal., 129 (1995), 355-383. MR.1361014 (96m:35216)

[19] J. Lenells, The Hunter-Saxton equation describes the geodesic flow on a sphere, J. Geom. Phys., 57 (2007), 2049-2064. MR2348278 (2008f:37169)

[20] J. Lenells, The Hunter-Saxton equation: a geometric approach, SIAM J. Math. Anal., 40 (2008), 266-277. MR 2403320 (2009b:37133)

[21] P. L. Lions, Mathematical Topics in Fluid Mechanics, Vol. I. Incompressible Models. Oxford Lecture Series in Mathematics and Applications, 3. Clarendon, Oxford University Press, New York, 1996. MR 1422251 (98b:76001)

[22] J. Liu and Z. Yin, Blow-up phenomena and global existence for a periodic Hunter-Saxton system, Preprint.

[23] M. V. Pavlov, The Gurevich-Zybin system, J. Phys. A: Math. Gen., 38 (2005), 3823-3840. MR2145381 (2005m:35241)

[24] P. Olver and P. Rosenau, Tri-Hamiltonian duality between solitons and solitary wave solutions having compact support, Phys. Rev. E (3), 53 (1996), 1900-1906. MR.1401317(97c:35172)

[25] M. Wunsch, On the Hunter-Saxton system, Discret Contin. Dyn. Syst. Ser. B, 12 (2009), 647-656. MR2525162 (2011a:35071)

[26] Z. Yin, On the structure of solutions to the periodic Hunter-Saxton equation, SIAM J. Math.Anal., 36 (2004), 272-283. MR2083862 (2005e:35034) 
[27] P. Zhang and Y. Zheng, On oscillations of an asymptotic equation of a nonlinear variational wave equation, Asymptot. Anal., 18 (1998), 307-327. MR.1668954 (99j:35146)

[28] P. Zhang and Y. Zheng, On the existence and uniqueness of solutions to an asymptotic equation of a nonlinear variational wave equation, Acta Math. Sinica, 15 (1999), 115-130. MR1701136 (2000f:35090)

[29] P. Zhang and Y. Zheng, Existence and uniqueness of solutions to an asymptotic equation from a variational wave equation with general data, Arch. Rat. Mech. Anal., 155 (2000), 49-83. MR1799274 (2001j:35184) 